\title{
The limit of discounted utilitarianism
}

\author{
AdAM Jonsson \\ Department of Engineering Sciences and Mathematics, Luleå University of Technology \\ MARK VOORNEVELD \\ Department of Economics, Stockholm School of Economics
}

\begin{abstract}
This paper presents an infinite-horizon version of intergenerational utilitarianism. By studying discounted utilitarianism as the discount factor tends to one, we obtain a new welfare criterion: limit-discounted utilitarianism (LDU). We show that LDU meets the standard assumptions of efficiency, equity, and interpersonal comparability, but allows us to compare more pairs of utility streams than commonly used utilitarian criteria, including the overtaking criterion and the catching-up criterion. We also introduce a principle of compensation for postponements of utility streams and use it to characterize the LDU criterion on a restricted domain.
\end{abstract}

KeYworDs. Utilitarianism, intergenerational equity, time preference.

JEL CLASSification. D63, D70, D90.

\section{INTRODUCTION}

Utilitarianism is the normative theory that asserts that the best social policy among a set of alternatives is the one that generates the greatest total welfare, where total welfare is often defined as the sum of the utilities for all members of society. This notion of maximizing aggregate utility becomes problematic for an infinite-horizon society. The problem of aggregating infinite utility streams $u=\left(u_{1}, u_{2}, \ldots\right)$, representing the utilities of present and future generations, has occupied philosophers and economists for more than a century. Discounted utilitarianism provides a popular criterion for the evaluation of infinite streams. However, since discounting means assigning smaller weights to future generations, discounted utilitarianism has also been the subject of heavy criticism. ${ }^{1}$ For instance, Ramsey (1928, p. 543) calls discounting future generations' utilities an "ethically indefensible" practice that "arises merely from the weakness of the imagination." In his axiomatic approach to discounted utilitarianism, Koopmans (1960) formally defines time preference through the concept of impatience. Subsequently, a long tradition

Adam Jonsson: adam.jonsson@ltu.se

Mark Voorneveld: mark . voorneveld@hhs . se

We thank two anonymous referees whose detailed comments led to substantial improvements. We also thank José Carlos Alcantud, Geir Asheim, David Borwein, Drew Fudenberg, Tapan Mitra, Malin Olsson, Marcus Pivato, Peter Wakker, and Jörgen Weibull for helpful discussions. Mark Voorneveld acknowledges financial support by the Wallander-Hedelius Foundation under grant P2010-0094:1.

${ }^{1}$ This criticism goes back at least to Sidgwick (1907) and Pigou (1920).

Copyright (C) 2018 The Authors. Theoretical Economics. The Econometric Society. Licensed under the Creative Commons Attribution-NonCommercial License 4.0. Available at http: //econtheory . org. https://doi.org/10.3982/TE1836 
in welfare economics studies social preferences that combine the Strong Pareto axiom with Anonymity, that is, invariance under transformations that swap the utility levels of any two generations. ${ }^{2}$ The formulation of criteria that meet the two requirements turns out to be associated with complications, the full extent of which has only been understood recently. First, on the set of infinite utility streams, complete and transitive preferences that satisfy Strong Pareto and Anonymity are not representable by social welfare functions. ${ }^{3}$ Second, such preferences cannot be obtained by constructive methods. In other words, all explicit descriptions of transitive preferences satisfying Strong Pareto and Anonymity are incomplete. ${ }^{4}$

The literature on intergenerational preferences satisfying Strong Pareto and Anonymity therefore uses incomplete preferences. A social welfare relation (SWR) is a binary relation that is reflexive and transitive, but not necessarily complete. The most often used utilitarian SWRs rank infinite streams on the basis of their partial sums over a finite horizon tending to infinity. ${ }^{5}$ This is the case, for instance, for the overtaking criterion (von Weizsäcker 1965), the catching-up criterion (Gale 1967), and the utilitarian SWR of Basu and Mitra (2007). ${ }^{6}$ This paper investigates an alternative approach. ${ }^{7}$ By studying discounted utilitarianism as the discount factor tends to one, we obtain a new welfare criterion: limit-discounted utilitarianism (LDU). In the limit, any two generations are treated equally, so this approach also avoids the critique of Ramsey (1928). Our main results include the following.

The Compensation Principle. Social welfare relations that satisfy the Anonymity axiom cannot exhibit impatience in the traditional sense of Koopmans (1960). ${ }^{8}$ However, in the literature stemming from his seminal contribution, many authors have argued for the appropriateness of a more inclusive concept of social time preference. The most common argument points out that the overtaking and catching-up criteria do not exhibit impatience in the sense of Koopmans $(1960)$, and rank $(1,0,1,0, \ldots)$ above

\footnotetext{
${ }^{2}$ Precise definitions of axioms from previous literature are in Section 2.

${ }^{3}$ Diamond's (1965) version of this result was obtained under an additional continuity assumption on the social welfare function. The general impossibility theorem, without this assumption, is due to Basu and Mitra (2003).

${ }^{4}$ The existence of complete, transitive binary relations satisfying Strong Pareto and Anonymity was established by Svensson (1980). Zame (2007, Theorem 4) and Lauwers (2010, p. 33) show that Svensson's existence theorem cannot be proved without using the Axiom of Choice.

${ }^{5}$ Formally, $\liminf _{T \rightarrow \infty} \sum_{t=1}^{T}\left(u_{t}-v_{t}\right) \geq 0$ must hold if $u=\left(u_{1}, u_{2}, \ldots\right)$ is at least as good as $v=\left(v_{1}, v_{2}, \ldots\right)$.

${ }^{6}$ The former would be more appropriately described as the SWRs induced by the overtaking and catching-up criteria. For convenience, the expressions SWR, criterion, and welfare criterion will be used synonymously.

${ }^{7}$ Discounting with discount factors tending to one has been used extensively in the literature on stochastic games and dynamic optimization; see, e.g., Liggett and Lippman (1969), Lippman (1969), Dutta (1991), Sennott (1999), and Bishop et al. (2014). Basu and Mitra (2007, pp. 360-361) defend the relevance of vanishing discount rates for intergenerational equity in a "robustness check" of their welfare criterion. They attribute the idea behind the robustness check to Jörgen Weibull. Limit-discounted utilitarianism can be seen as a concretization of their line of thought.

${ }^{8}$ Koopmans's definition (see Koopmans 1960, Def. 1) is stated for pre-orders defined by social welfare functions. According to Banerjee and Mitra's (2007, Section 2.2.2) more general ordinal formulation, impatience is displayed by strictly preferring a stream $u$ with $u_{s}>u_{t}$ for some $s<t$ to the stream $v$ obtained by switching $u_{s}$ and $u_{t}$. With Anonymity, the two streams are equivalent.
} 
$(0,1,0,1, \ldots)$; see, for example, Lauwers (1995), Fleurbaey and Michel (2003), and Heal (2005). This literature advocates anonymity axioms involving classes of infinite permutations that make $(1,0,1,0, \ldots)$ equivalent to $(0,1,0,1, \ldots)$. Recently, several authors (including Dutta (2008) and Asheim et al. (2010)) have remarked that the equivalence of the two periodic streams means a violation of either Strong Pareto or Stationarity, two often combined conditions in intertemporal contexts. The Compensation Principle addresses an aspect of time preference-the effect of postponing utility streams-in a way that does not conflict with these conditions. Informally, the principle says that a utility stream can be postponed for one generation if the infinite-horizon society is compensated by the average utility over all generations. The precise formulation is in Section 2, where we also motivate the average as the suitable compensation. If $u=(1,0,1,0, \ldots)$, the Compensation Principle says that $u$ is equivalent to $(1 / 2, u)$, which makes $u$ strictly preferred to $(0, u)=(0,1,0,1, \ldots)$ by transitivity and Strong Pareto. In short, the Compensation Principle provides a new perspective on time preference in a widely discussed collection of examples.

A characterization. Theorem 1 shows that Strong Pareto, the Compensation Principle, and Additivity (a standard translation invariance axiom) characterize limitdiscounted utilitarianism on the set of pairs of utility streams with a summable or eventually periodic difference.

Basic properties. Theorem 2 shows that besides Strong Pareto and Additivity, the limit-discounted utilitarian criterion satisfies Anonymity, Stationarity, and a continuity property relating preferences over infinite streams to preferences over long, finitehorizon truncations. This continuity requirement is a relaxation of a similar requirement in Brock's (1970) classical characterization of the overtaking criterion. Theorem 2 also shows that LDU meets the utilitarian requirement that summable streams with a larger sum are strictly preferred to those with a smaller sum; streams with equal finite sums are equivalent.

Comparison to other utilitarian criteria. The limit-discounted utilitarian criterion is closely related to the Abel summation method from the theory of divergent series. In Lemma 1, we generalize the classical theorem of Frobenius (1880) on the relationship between Abel and Cesàro summation to obtain a sufficient condition for comparability. Theorem 3 summarizes relations between our criterion and overtaking, catchingup, and the Basu-Mitra criterion: If $u$ is weakly preferred to $v$ according to any of these three criteria, then the same is true for LDU. For the Basu-Mitra criterion, the implication holds for strict preference as well. Example 2 contains a class of streams that can be ranked with LDU, but cannot be ranked using overtaking, catching-up, or the Basu-Mitra criterion. Finally, Example 4 provides a pair of streams that LDU does not compare.

\section{INTRODUCING THE LIMIT-DISCOUNTED UTILITARIAN CRITERION}

This section formally introduces our notion of limit-discounted utilitarianism and gives an axiomatic description of the limit-discounted utilitarian criterion. We also show that our criterion has a number of properties that are familiar from earlier literature. 
The following notation will be used: $\mathbb{N}=\{1,2,3, \ldots\}$ is the set of positive integers, $\mathbb{R}$ is the set of real numbers, and

$$
\mathcal{U}=\left\{u \in \mathbb{R}^{\mathbb{N}}: \sup _{t \in \mathbb{N}}\left|u_{t}\right|<+\infty\right\}
$$

is the set of bounded utility streams $u=\left(u_{1}, u_{2}, \ldots\right)$, where $u_{t}$ denotes the welfare of generation $t \in \mathbb{N}$. A social welfare relation (SWR) is a reflexive and transitive binary relation $\succsim$ on $u$. For $u, v \in \mathcal{U}, u \succsim v$ means that society considers $u$ to be at least as good as $v$. As usual, $u \sim v$ means that $u \succsim v$ and $v \succsim u$, whereas $u \succ v$ means that $u \succsim v$ but not $v \succsim u$.

Given $u \in U$, the Abel sum

$$
\lim _{\delta \rightarrow 1^{-}} \sum_{t=1}^{\infty} \delta^{t-1} u_{t}
$$

will be denoted by $\sigma_{A}(u)$ if the limit (1) exists and is finite. It is easy to see that this limit, if it exists, is unaffected by switching any two entries of $u$. Abel summation thus provides a method for evaluating streams using discounting while treating each pair of generations equally. Our concept of limit-discounted utilitarianism builds on Abel summation and is formally defined as follows:

Definition 1. The limit-discounted utilitarian (LDU) criterion is defined for all $u, v \in$ $u$ as

$$
u \succsim_{\mathrm{LDU}} v \Longleftrightarrow \liminf _{\delta \rightarrow 1^{-}} \sum_{t=1}^{\infty} \delta^{t-1}\left(u_{t}-v_{t}\right) \geq 0 .
$$

Our first objective is to give an axiomatic description of the LDU criterion. We do so on a domain that accommodates frequently discussed examples from recent literature, including all pairs $u, v \in \mathcal{U}$ of eventually periodic streams, namely, ${ }^{9}$

$$
\mathcal{D} \equiv\{(u, v) \in \mathcal{U} \times u: u-v \text { is summable or eventually periodic }\} .
$$

Our characterization result uses three properties of LDU. We show that for a SWR $\succsim$ on $u$ with these three properties, every pair of streams in $\mathcal{D}$ can be compared using $\succsim$, where $u \succsim v$ holds if and only if $u \succsim_{\text {LDU }} v$. The first two properties are the traditional assumptions of efficiency and interpersonal comparisons of utility, respectively:

Strong Pareto (SP). For all $u, v \in \mathcal{U}$, if $u_{t} \geq v_{t}$ for all $t \in \mathbb{N}$ and $u \neq v$, then $u \succ v$.

Additivity (Add). For all $u, v, \alpha \in \mathcal{U}, u \succsim v$ implies $u+\alpha \succsim v+\alpha$.

\footnotetext{
${ }^{9}$ Stream $u \in U$ is summable if the series $\sum_{t=1}^{\infty} u_{t}$ converges, and is eventually periodic (with period $p$ ) if there are $k, p \in \mathbb{N}$ with $u_{t+p}=u_{t}$ for all $t \geq k$.
} 
Several authors use assumptions on interpersonal comparisons that are weaker than Additivity. ${ }^{10}$ But since $u-v=(u+\alpha)-(v+\alpha)$ for all $u, v, \alpha \in U$, Additivity is satisfied if weak preference $u \succsim v$ depends only on $u-v$. This is the case for all SWRs in this paper.

The third property is a principle of compensation for postponements of infinite utility streams. Given $u \in U$ and $c \in \mathbb{R}$, call $(c, u) \equiv\left(c, u_{1}, u_{2}, \ldots\right)$ the postponement of $u \in U$ with compensation $c .{ }^{11}$ The Compensation Principle says that if the average $\bar{u} \equiv \lim _{n \rightarrow \infty} \sum_{t=1}^{n} u_{t} / n$ of $u \in U$ is well defined, then $c=\bar{u}$ is a suitable compensation for a postponement of $u$.

Compensation Principle (CP). For all $u \in U$, if $\bar{u}$ is well defined, then

$$
(\bar{u}, u) \sim u
$$

Notice that if SWR $\succsim$ satisfies Strong Pareto and $u=(a, a, a, \ldots)$ is constant, then $u \succ$ $(c, u)$ if $c<a$. In this case, $(c, u) \sim u$ can only hold if $c=a$, i.e., the average of $u$. Is there a more general rationale for this compensation level? We give an answer to this question below: commonly used assumptions on intertemporal preferences allow us to conclude that $(c, u) \sim u$ implies $c=\bar{u}$ on a larger set of streams. But let us first observe that the three properties characterize LDU on $\mathscr{D}$. All proofs are provided in the Appendix.

Theorem 1. The binary relation $\succsim_{\mathrm{LD}}$ defines a SWR that satisfies Strong Pareto, Additivity, and the Compensation Principle. Every SWR on $U$ with these three properties coincides with $\succsim_{\text {LDU }}$ on D .

We sketch the proof of this theorem. For the second assertion, let $\succsim$ be a SWR on $u$ with the three properties. The key observation is that given any $u, v \in \mathcal{U}$, the sequence $s=\left(s_{1}, s_{2}, \ldots\right)$ of partial sums $s_{n}=\sum_{t=1}^{n}\left(u_{t}-v_{t}\right)$ satisfies $u-v=s-(0, s) .{ }^{12}$ By Additivity, this means that if $s$ is bounded (so that $s$ and $(0, s)$ are both in $u$ ), then $u \succsim v$ holds if and only if $s \succsim(0, s)$. Suppose, in addition, that $\bar{s}$ is well defined. We then have $(\bar{s}, s) \sim s$ by the Compensation Principle. Using Additivity and Strong Pareto, we are able to conclude ((14)-(15)) that

$$
u \succsim v \Longleftrightarrow \bar{s} \geq 0
$$

The average of $s$ is, by definition, the Cesàro sum of the series $\sum_{t=1}^{\infty}\left(u_{t}-v_{t}\right)$. By the theorem of Frobenius (see Duren 2012, p. 180), the series is Abel-summable to the same sum:

$$
\sigma_{A}(u-v)=\bar{s} .
$$

\footnotetext{
${ }^{10}$ These include the Partial Unit Comparability axiom in Basu and Mitra (2007) and the Partial and Finite Translation Scale Invariance axiom in Asheim (2010) and Asheim et al. (2010), respectively.

${ }^{11}$ Heal (2005, p. 1115) refers to $(0, u)$ as the postponement of $u$. Koopmans et al. (1964) refer to $(c, u)$ as the postponement of $u$ with "insertion" $c$.

${ }^{12}$ That is, for each $t, s_{t+1}-s_{t}=u_{t+1}-v_{t+1}$.
} 
Combining (2) and (3) gives

$$
u \succsim v \Longleftrightarrow u \succsim_{\mathrm{LDU}} v
$$

This argument takes care of the case when $d=u-v$ is summable or eventually periodic with average $\bar{d}=0$ (Case 1 in the proof), as $\bar{s}$ is defined in this case. A monotonicity argument reduces the case when $\bar{d} \neq 0$ (Case 2) to the zero-average case.

Let us note that (3) gives us an interpretation of the Compensation Principle in terms of the loss that society incurs when a stream is postponed with $c=0$. To elaborate, suppose $u \in U$ has a well defined average and let $v=(0, u)$. We then have $s_{n}=\sum_{t=1}^{n}\left(u_{t}-v_{t}\right)=u_{n}$ for all $n \geq 1$, so the partial sums have average $\bar{s}=\bar{u}$, which by (3) means that $\sigma_{A}(u-v)=\bar{u}$. In other words, in terms of Abel summation, postponing $u$ with compensation $c=0$ incurs a loss of $\bar{u}$ whenever $\bar{u}$ is well defined. For example, postponing $u=(1,1,1, \ldots)$ incurs a loss of $\bar{u}=1$ and postponing $w=(1,0,1,0, \ldots)$ incurs a loss of $\bar{w}=1 / 2$.

We now turn to the properties mentioned in the Introduction. These properties will not only help us relate LDU to established utilitarian SWRs, which is the topic of the next section. They will also allow us to provide axiomatic support for the Compensation Principle. The Anonymity axiom says that preferences are unaffected by switching the utility levels of any two generations.

Anonymity (Ano). For all $u, v \in \mathcal{U}$, if there are $s, t \in \mathbb{N}$ with $u_{s}=v_{t}$ and $u_{t}=v_{s}$, and if $u_{i}=v_{i}$ for all other $i \in \mathbb{N}$, then $u \sim v$.

Stationarity is Koopmans's (1960) condition for dynamic consistency: preferences are independent of the first generation if the first generation receives identical utility levels in the social states specified by $u$ and $v .^{13}$

Stationarity (Stat). For all $u, v \in U$ and $c \in \mathbb{R}, u \succsim v$ if and only if $(c, u) \succsim(c, v)$.

The compensation level in the Compensation Principle can in part be justified by Stationarity and the following property.

Total Utility (TU). For all $u, v \in \mathcal{U}$, if $u-v$ is summable, then

$$
u \succsim v \text { if and only if } \sum_{t=1}^{\infty}\left(u_{t}-v_{t}\right) \geq 0 .
$$

If $\succsim$ is a stationary SWR with this property and $u$ is eventually periodic, then $(c, u) \sim u$ can only hold if $c=\bar{u}$. To illustrate the argument with a simple example, let $u=(a, b, a, b, \ldots)$, so that $u$ is periodic with period 2. If $(c, u) \sim u$, then we have $(c, c, u) \sim(c, u)$ by Stationarity and, hence,

$$
(c, c, u) \sim u
$$

\footnotetext{
${ }^{13}$ Also Asheim et al. (2010) stress the relevance of Stationarity for intergenerational utilitarianism. They motivate their extension of Basu and Mitra's relation (8) by the desirability of retaining this axiom.
} 
by transitivity. Since $(c, c, u)-u=(c-a, c-b, 0,0, \ldots)$, combining (4) and (5) gives $(c-a)+(c-b)=0$, i.e., $c=(a+b) / 2$, the average of $u=(a, b, a, b, \ldots)$. Here we only needed to assume that (4) holds when $u-v$ has at most a finite number of nonzero entries, and for this it is enough to impose Strong Pareto, Additivity, and Anonymity. ${ }^{14}$ So if we add Stationarity to this axiom set, then $(c, u) \sim u$ implies $c=\bar{u}$ for all eventually periodic $u$ (Proposition 1 in the Appendix).

This implication (if $(c, u) \sim u$, then $c=\bar{u}$ ) holds on a larger set of streams, including all convergent and, consequently, all summable streams, if we add a continuity assumption to the axioms above. We defer the precise definition of this set of streams to the Appendix. The continuity assumption in question relates preferences over infinite streams to preferences over long, finite-horizon truncations: for $u \in U$ and $n \in \mathbb{N}$, we write $u_{[n]}=\left(u_{1}, u_{2}, \ldots, u_{n}, 0,0, \ldots\right)$ and define ${ }^{15}$ the following axiom.

Continuity (Cont). For all $u, v \in \mathcal{U}$, if there is an $N \in \mathbb{N}$ with $u_{[n]} \succ v_{[n]}$ for all $n \geq N$, then $u \succsim v$.

A similar axiom is used by Brock (1970), who writes that his condition captures the idea that "decisions on infinite programs are consistent with decisions on finite programs of length $n$ if $n$ is large enough." For a discussion of the two axioms and their relation to the Total Utility property, see Jonsson and Voorneveld (2015).

Theorem 2. The LDU criterion satisfies Anonymity, Stationarity, Total Utility, and Continuity.

The first three properties in Theorem 2 are easily verified. Anonymity is implied by the Total Utility property, which in turn is a direct consequence of Abel's theorem. To verify that LDU satisfies Continuity, we use a generalization of the Frobenius theorem that relates upper and lower Cesàro and Abel limits. ${ }^{16}$ For $d \in U$ and $\delta \in(0,1)$, define $s_{n}=\sum_{t=1}^{n} d_{t}, n \geq 1$, and

$$
\begin{aligned}
& C_{n}(d)=\frac{s_{1}+s_{2}+\cdots+s_{n}}{n}, \\
& \sigma_{\delta}(d)=\sum_{t=1}^{\infty} \delta^{t-1} d_{t} .
\end{aligned}
$$

Many properties of the LDU criterion are consequences of the following result.

\footnotetext{
${ }^{14}$ See Basu and Mitra (2007, Lemma 1) or Jonsson and Voorneveld (2015, Lemma 1).

${ }^{15}$ Our convention of considering streams with $u_{t}=0$ for $t \geq n$ follows Brock (1970) and Basu and Mitra (2007). With Additivity, replacing the zeros by any other numbers would give the same condition as long as generations $t>n$ receive the same welfare in the two social states. For instance, if Additivity is satisfied, then Continuity is equivalent to a relaxation of the "weak preference continuity" axiom in Asheim and Tungodden (2004, p. 223): for all $u, v \in U$, if there is an $N \in \mathbb{N}$ with $\left(u_{1}, \ldots, u_{n}, v_{n+1}, v_{n+2}, \ldots\right) \succ v$ for all $n \geq N$, then $u \succsim v$.

${ }^{16}$ The inequalities in Lemma 1 are well known from the literature on stochastic games; see Lippman (1969), Bishop et al. (2014), and Sennott (1999). These references do not contain a proof of the result in the generality that we stated it. Our proof is a slightly rewritten version of one suggested by an anonymous referee.
} 
Lemma 1. For $d \in \mathcal{U}$,

$$
\liminf _{n \rightarrow \infty} C_{n}(d) \leq \liminf _{\delta \rightarrow 1^{-}} \sigma_{\delta}(d) \leq \limsup _{\delta \rightarrow 1^{-}} \sigma_{\delta}(d) \leq \limsup _{n \rightarrow \infty} C_{n}(d) .
$$

This result will also help us in the next section when we compare our criterion to the overtaking criterion and the catching-up criterion.

\section{COMPARISON TO OTHER UTILITARIAN CRITERIA}

In this section, we compare the limit-discounted utilitarian criterion to three other utilitarian social welfare relations: ${ }^{17}$ the criterion $\succsim_{\text {вм }}$ of Basu and Mitra (2007), where

$$
\begin{gathered}
u \succsim_{\text {вм }} v \Longleftrightarrow \quad \text { there is a } T_{0} \in \mathbb{N} \text { with } \sum_{t=1}^{T_{0}}\left(u_{t}-v_{t}\right) \geq 0 \\
\text { and } u_{t}-v_{t} \geq 0 \text { for all } t \geq T_{0},
\end{gathered}
$$

the overtaking criterion ${ }_{\mathrm{w}}$ of von Weizsäcker (1965), where

$$
u \succsim_{\mathrm{w}} v \quad \Longleftrightarrow \quad \text { there is a } T_{0} \in \mathbb{N} \text { with } \sum_{t=1}^{T}\left(u_{t}-v_{t}\right) \geq 0 \text { for all } T \geq T_{0} \text {, }
$$

and the catching-up criterion $\succsim_{\mathrm{G}}$ of Gale (1967), where

$$
u \succsim_{\mathrm{G}} v \Longleftrightarrow \liminf _{T \rightarrow \infty} \sum_{t=1}^{T}\left(u_{t}-v_{t}\right) \geq 0 .
$$

Our discussion addresses the following (related) questions: Which properties from the previous section do these criteria satisfy, and to what extent do preferences according to LDU agree with those of the other criteria?

Table 1 lists the properties of LDU established in the previous section and indicates which of these properties are satisfied by overtaking, catching-up, and the Basu-Mitra criterion.

The properties that are satisfied by the three criteria are straightforward to verify and are known from the literature. ${ }^{18}$ We illustrate violations of properties in two examples where the overtaking criterion $\succsim_{W}$ and the catching-up criterion $\succsim_{\mathrm{G}}$ have been criticized. ${ }^{19}$

\footnotetext{
${ }^{17}$ Our definitions of the overtaking and catching-up criteria follow Gale (1967).

${ }^{18}$ See Basu and Mitra (2007) and Jonsson and Voorneveld (2015).

${ }^{19}$ Basu and Mitra (2007, p. 361) consider a version of Example 1. Versions of Example 2 have been discussed by, among others, Lauwers (1995, p. 348), Lauwers (1997, p. 225), Fleurbaey and Michel (2003, p. 783), Asheim and Tungodden (2004, p. 229), Heal (2005, p. 1115), Banerjee (2006, p. 333), Basu and Mitra (2007, p. 360), Dutta (2008, Sec. IV), Asheim et al. (2010, p. 520), and Asheim and Banerjee (2010, p. 164).
} 


\begin{tabular}{llccccccc}
\hline & & SP & Add & CP & Ano & Stat & Cont & TU \\
\hline LDU & $\succsim_{\text {LDU }}$ & + & + & + & + & + & + & + \\
Basu-Mitra & $\succsim_{\text {BM }}$ & + & + & - & + & + & - & - \\
Overtaking & $\succsim_{\mathrm{W}}$ & + & + & - & + & + & + & - \\
Catching-up & $\succsim_{\text {G }}$ & + & + & - & + & + & + & + \\
\hline
\end{tabular}

TABLE 1. Social welfare relations and properties they do (+) or do not (-) satisfy.

EXAMPLE 1. Let $u \in U$ be a summable stream with strictly positive entries and let $v=$ $(0, u)$. Then $\sum_{t=1}^{T}\left(u_{t}-v_{t}\right)=u_{T}>0$ for all $T \geq 1$. So $u$ is strictly preferred to $v=(0, u)$ under overtaking. Since the two streams have the same sum, this example illustrates that overtaking does not have the Total Utility property; neither does the Basu-Mitra criterion, which cannot compare $u$ and $v .^{20}$ LDU and catching-up do have the Total Utility property: they find $u$ and $(0, u)$ equivalent.

The next example shows that the Compensation Principle and Strong Pareto allow us to compare streams that have appeared frequently in the literature, but that are not comparable using overtaking, catching-up, or the Basu-Mitra criterion.

Example 2. Consider the periodic stream $u=(1,0,1,0, \ldots)$ and let $v=(c, u)$ for some real number $c$. Since $u-v=(1-c,-1,1,-1, \ldots)$, Basu and Mitra's criterion does not compare $u$ and $v$ for any value of $c$. We have

$$
\sum_{t=1}^{T}\left(u_{t}-v_{t}\right)=-\sum_{t=1}^{T}\left(v_{t}-u_{t}\right)= \begin{cases}-c+1 & \text { if } T \text { is odd } \\ -c & \text { if } T \text { is even }\end{cases}
$$

So $u$ and $v$ are not comparable with the overtaking criterion or the catching-up criterion if $c \in(0,1)$. Both criteria rank $u$ above $v$ if $c \leq 0$. In particular, they prefer $(1,0,1,0, \ldots)$ to $(0,1,0,1, \ldots)$. The LDU criterion compares $u$ and $v$ for all $c$. Indeed, for $\delta \in(0,1)$,

$$
\sum_{t=1}^{\infty} \delta^{t-1}\left(u_{t}-v_{t}\right)=\sum_{t=1}^{\infty}(-\delta)^{t-1}-c=\frac{1}{1+\delta}-c .
$$

Letting $\delta$ go to 1 , the Abel sum of $\sum_{t=1}^{\infty}\left(u_{t}-v_{t}\right)$ is $1 / 2-c$. This means that $u \sim_{\text {LDU }} v$ if $c=1 / 2, u \succ_{\text {LDU }} v$ if $c<1 / 2$, and $v \succ_{\text {LDU }} u$ if $c>1 / 2$. From the axioms, we have $u \sim_{\text {LDU }} v$ for $c=1 / 2$ by the Compensation Principle and, therefore, strict preference for all other $c$ by Strong Pareto.

The next example contains a pair of streams over which catching-up has a strict preference, but LDU is indifferent; Example 1 gave a corresponding result for overtaking.

\footnotetext{
${ }^{20}$ Since $\sum_{t=1}^{\infty} u_{t}=\sum_{t=1}^{\infty} v_{t}$ and $\sum_{t=1}^{T}\left(u_{t}-v_{t}\right)>0$ for every $T$, there is no $T_{0}$ with $u_{t} \geq v_{t}$ for all $t \geq T_{0}$. Therefore, $u \succsim_{\text {вм }} v$ does not hold. Likewise, $v \succsim_{\text {вм }} u$ does not hold. This also shows that $\succsim_{\text {вм }}$ violates Continuity: $u_{[n]} \succ_{\mathrm{BM}} v_{[n]}$ for all $n$, but $u$ and $v$ are not comparable.
} 
Example 3. Define $u \in U$ by setting $u_{t}=1$ if $t=2^{n}$ for some $n \in \mathbb{N}$ and $u_{t}=0$ otherwise. Then $u-(0, u)=\left(u_{1}, u_{2}-u_{1}, u_{3}-u_{2}, \ldots\right)$ has partial sums $s_{n}=u_{n}$, which means that $u \succ_{\mathrm{G}}(0, u)$. But since $\bar{u}=0$, the Compensation Principle implies that $u \sim_{\mathrm{LDU}}(0, u)$.

To describe to what degree LDU agrees with overtaking, catching up, and the BasuMitra criterion, we use the following terminology: Given two SWRs $\succsim_{A}$ and $\succsim_{B}$ on $\mathcal{U}$, say that $\succsim_{B}$ weakly extends $\succsim_{A}$ if for all $u, v \in \mathcal{U}, u \succsim_{A} v$ implies $u \succsim_{B} v$. If, in addition, for all $u, v \in U, u \succ_{A} v$ implies $u \succ_{B} v$, we say that $\succsim_{B}$ extends $\succsim_{A}$.

THEOREм 3. The following relations hold between the LDU criterion $\succsim_{\mathrm{LDU}}$, the BasuMitra criterion $\succsim_{\mathrm{BM}}$, overtaking $\succsim_{\mathrm{W}}$, and catching- $u \mathrm{p} \succsim_{\mathrm{G}}$ :

(i) $\succsim_{\mathrm{LDU}}$ extends $\succsim_{\mathrm{BM}}$

(ii) $\succsim_{\mathrm{LDU}}$ weakly extends $\succsim_{\mathrm{G}}$ and $\succsim_{\mathrm{G}}$ weakly extends $\succsim_{\mathrm{W}}$

(iii) $\succsim_{\mathrm{LDU}}$ does not extend $\succsim_{\mathrm{w}}$ : there are $u, v \in \mathcal{U}$ with $u \succ_{\mathrm{W}} v$ and $u \sim_{\mathrm{LDU}} v$

(iv) $\succsim_{\mathrm{LDU}}$ does not extend $\succsim_{\mathrm{G}}$ : there are $u, v \in \mathcal{u}$ with $u \succ_{\mathrm{G}} v$ and $u \sim_{\mathrm{LDU}} v$.

In summary, if a pair of streams can be compared using overtaking, catching-up, or the Basu-Mitra criterion, then the same is true for LDU. But we also saw that LDU can compare streams that have appeared frequently in the literature, but that are not comparable using any of these other criteria. The pairs of streams in Examples 1 and 2 are all in $\mathscr{D}$. Theorem 1 shows that all pairs of streams in this domain can be compared using Additivity, Strong Pareto, and the Compensation Principle.

\section{Limitations}

We conclude our study of the LDU criterion by providing examples of streams that it does not allow us to rank. By means of these examples, we briefly discuss the extent to which LDU is characterized by properties that we have established.

Lemma 1 tells us that for all $u, v \in \mathcal{U}$,

$$
\liminf _{\delta \rightarrow 1^{-}} \sigma_{\delta}(u-v) \geq \liminf _{n \rightarrow \infty} C_{n}(u-v) .
$$

If the partial sums $\sum_{t=1}^{n}\left(u_{t}-v_{t}\right), n \geq 1$, are bounded below or above and $\lim _{\delta \rightarrow 1^{-}} \sigma_{\delta}(u-v)$ exists, then the inequality in (9) is an equality. ${ }^{21}$ That we may have strict inequality in (9) even with bounded partial sums was demonstrated by, among others, Liggett and Lippman (1969). We use an example from Bishop et al. (2014, Section 4 ), to define a pair of streams that $\succsim_{\text {LDU }}$ does not compare.

Example 4. Define $a=\left(a_{1}, a_{2}, \ldots\right) \in \mathcal{U}$ by

$$
a_{t}= \begin{cases}0 & \text { if } k ! \leq t<2 k ! \text { for some } k \in \mathbb{N} \\ 1 & \text { otherwise. }\end{cases}
$$

\footnotetext{
${ }^{21}$ This follows from the Hardy-Littlewood theorem (Duren 2012, p. 184).
} 
We then have (Bishop et al. 2014, Proposition 2)

$$
\liminf _{n \rightarrow \infty} \frac{1}{n} \sum_{t=1}^{n} a_{t}=1 / 2<\liminf _{\delta \rightarrow 1^{-}}(1-\delta) \sigma_{\delta}(a)=3 / 4
$$

and

$$
\limsup _{n \rightarrow \infty} \frac{1}{n} \sum_{t=1}^{n} a_{t}=\limsup _{\delta \rightarrow 1^{-}}(1-\delta) \sigma_{\delta}(a)=1
$$

If we take $u=a-(0, a)$, then the partial sums of $u$ equal $s_{n}=\sum_{t=1}^{n} u_{t}=a_{n}, n \geq 1$. Summation by parts gives that $\sigma_{\delta}(u)=(1-\delta) \sigma_{\delta}(a)$. By (10) and (11),

$$
\liminf _{n \rightarrow \infty} C_{n}(u)=1 / 2<\liminf _{\delta \rightarrow 1^{-}} \sigma_{\delta}(u)=3 / 4 \text { and } \quad \limsup _{n \rightarrow \infty} C_{n}(u)=\limsup _{\delta \rightarrow 1^{-}} \sigma_{\delta}(u)=1 .
$$

Consequently, $u$ and $v=(r, 0,0, \ldots)$ are not $\succsim_{\mathrm{LDU}}$-comparable if $r \in(3 / 4,1)$.

The arguments in the proof of Theorem 1 apply whenever the sequence $s=$ $\left(s_{1}, s_{2}, \ldots\right)$ of partial sums $s_{n}=\sum_{t=1}^{n}\left(u_{t}-v_{t}\right), n \geq 1$, is bounded and has a well defined average. But if $\bar{s}$ is undefined, then the Compensation Principle does not help us compare $s$ and $(0, s)$. Lemma 1 gives us that for all $u \in U$ and $c \in \mathbb{R},(c, u) \succsim_{\text {LDU }} u$ if $c \geq \lim \sup _{n \rightarrow \infty} \frac{1}{n} \sum_{t=1}^{n} u_{t}$, where $u \succsim_{\mathrm{LDU}}(c, u)$ if $c \leq \liminf _{n \rightarrow \infty} \frac{1}{n} \sum_{t=1}^{n} u_{t}$. However, characterizing LDU on larger domains is made difficult by the complicated relations between upper and lower Abel and Cesàro limits (see Bishop et al. 2014 and Example 4 above). We leave this investigation for future work.

\section{APPENDiX}

This appendix contains all proofs. Proposition 1 in Section A.1 gives axiomatic support for using the average in our Compensation Principle. Sections A.2-A.5 contain proofs of Theorem 1, Lemma 1, Theorem 2, and Theorem 3, respectively. In the proofs, we refer to axioms using their abbreviations from Section 2.

\section{A.1 Motivating the Compensation Principle}

We argue that if a stream $u$ on average gives each generation a utility of $\bar{u}$, then $c=\bar{u}$ is a reasonable compensation in a compensated postponement $(c, u)$ of $u$ : using some of our earlier axioms, Proposition 1 shows-on a fairly large set of well behaved streamsthat $(c, u) \sim u$ can only hold if $c=\bar{u}$. We say that a stream $u \in U$ has a regular average if (i) its average $\bar{u}$ is well defined and (ii) for every $\varepsilon>0$, the average of $u$ over sufficiently long, but finite, segments of consecutive coordinates differs from $\bar{u}$ by at most $\varepsilon$. That is, $u \in \mathcal{U}$ has a regular average if $\bar{u}$ is well defined and for every $\varepsilon>0$, there is an $N \in \mathbb{N}$ such that

$$
\left|\frac{1}{n} \sum_{t=t_{0}+1}^{t_{0}+n} u_{t}-\bar{u}\right|<\varepsilon \quad \text { for all } t_{0} \in \mathbb{N} \text { and } n \geq N
$$


For instance, the set of streams with a regular average contains all streams that are eventually periodic, summable, or convergent. ${ }^{22}$

Proposition 1. Let $\succsim$ be a SWR on U that satisfies Strong Pareto, Anonymity, Additivity, and Stationarity. If $u \in U$ is eventually periodic and $c \in \mathbb{R}$, then

(i) $(c, u) \succsim u$ implies $c \geq \bar{u}$

(ii) $u \succsim(c, u)$ implies $c \leq \bar{u}$

(iii) $(c, u) \sim u$ implies $c=\bar{u}$.

If $\succsim$ also satisfies Continuity, then these implications hold for all $u \in \mathcal{U}$ with a regular average.

Proof. Recall from footnote 14 that SP, Ano, and Add imply that for all $u, v \in \mathcal{U}$, where $u-v$ has only finitely many nonzero entries,

$$
u \succsim v \quad \Longleftrightarrow \quad \sum_{t=1}^{\infty}\left(u_{t}-v_{t}\right) \geq 0 .
$$

Assume that SWR $\succsim$ on $U$ satisfies SP, Ano, Add, and Stat. Let $u \in U$ be eventually periodic and let $c \in \mathbb{R}$. To prove (i), assume that $(c, u) \succsim u$. Since $u$ is eventually periodic, there are $k, p \in \mathbb{N}$ with $u_{t+p}=u_{t}$ for $t \geq k$. If $p=1$, then $u=\left(u_{1}, \ldots, u_{k}, u_{k}, u_{k}, \ldots\right)$ is eventually constant, so $(c, u)-u$ has a finite number of nonzero entries that sum to $c-u_{k}$. Since SP, Ano, and Add are satisfied, (12) implies that $c \geq u_{k}=\bar{u}$. If $p \geq 2$, we have $(c, c, u) \succsim(c, u) \succsim u$ by Stat and $(c, c, u) \succsim u$ by transitivity. Iterating (if $p>2$ ) gives $\left(\underline{c}_{p}, u\right) \succsim u$, where $\underline{c}_{p}$ is a shortcut notation for $p$ consecutive coordinates equal to $c$. Since $u_{t}=\left(\underline{c}_{p}, u\right)_{t}$ for all $t>k+p$, the difference $\left(\underline{c}_{p}, u\right)-u$ has finitely many nonzero entries and sum $p c-\sum_{t=k+1}^{k+p} u_{t}$. By (12), $\left(\underline{c}_{p}, u\right) \succsim u$ implies that that this sum is nonnegative: $c \geq \sum_{t=k+1}^{k+p} u_{t} / p=\bar{u}$.

(ii) By Add, $u \succsim(c, u)$ implies $(-c,-u) \succsim-u$, which by (i) implies $-c \geq-\bar{u}$, so that $c \leq \bar{u}$.

(iii) This follows from (i) and (ii).

Now add axiom Cont. We prove implication (i) for streams with a regular average; (ii) and (iii) follow as above. So let $u \in \mathcal{U}$ and $c \in \mathbb{R}$ be such that $u$ has regular average $\bar{u}$ and $(c, u) \succsim u$. By Stat and transitivity, $\left(\underline{c}_{N}, u\right) \succsim u$ for all $N \in \mathbb{N}$. To prove that $c \geq \bar{u}$, suppose, to the contrary, that $c<\bar{u}$. We obtain a contradiction by showing that $u \succ\left(\underline{c}_{N}, u\right)$ for some $N \in \mathbb{N}$.

If $c<\bar{u}$, there are $b \in \mathbb{R}$ with $c<b<\bar{u}$ and $N \in \mathbb{N}$ such that the average of $u$ over any $n \geq N$ consecutive generations differs from $\bar{u}$ by at most $\varepsilon \equiv(\bar{u}-b) / 2$. Let

\footnotetext{
${ }^{22} \mathrm{~A}$ stream with an average that is not regular is $u=(0,1,0,0,1,1,0,0,0,1,1,1, \ldots)$, consisting of a 0 followed by a 1 , then two 0 s followed by two 1 s, three 0 s followed by three 1 s, etc. Its average is $1 / 2$. But it does not have a regular average: for every $n$, the stream contains infinitely many segments of $n$ consecutive 0 s (and $n$ consecutive $1 \mathrm{~s}$ ).
} 
$d=u-\left(\underline{b}_{N}, u\right)$. For $n>N$,

$$
\begin{aligned}
\sum_{t=1}^{n} d_{t} & =u_{n}+u_{n-1}+\cdots+u_{n-N+1}-N b \\
& =N\left(\left(u_{n}+u_{n-1}+\cdots+u_{n-N+1}\right) / N-b\right) \\
& \geq N(\bar{u}-\varepsilon-b) \\
& =N \varepsilon .
\end{aligned}
$$

Since $d_{[n]}$ has finitely many nonzero entries and positive sum, (12) implies that $d_{[n]} \succ$ $(0,0,0, \ldots)$ for all $n>N$. By Cont, we have $d \succsim(0,0,0, \ldots)$. Hence $u \succsim\left(\underline{b}_{N}, u\right)$ by Add. By SP and $b>c, u \succ\left(\underline{c}_{N}, u\right)$, which is our contradiction.

\section{A.2 Proving Theorem 1}

We start with a lemma linking Strong Pareto, Additivity, and the Compensation Principle to summability.

Lemma 2. Let the SWR $\succsim$ satisfy Strong Pareto, Additivity, and the Compensation Principle. For $u, v \in U$, if the series $\sum_{t=1}^{\infty}\left(u_{t}-v_{t}\right)$ is Cesàro-summable and has bounded partial sums, then

$$
u \succsim v \Longleftrightarrow \lim _{n \rightarrow \infty} C_{n}(u-v) \geq 0 .
$$

In particular, $\succsim$ has the Total Utility property.

Proof. For $u$ and $v$ as in the lemma, Add gives $u \succsim v$ if and only if $u-v \succsim(0,0, \ldots)$. The sequence $s=\left(s_{1}, s_{2}, \ldots\right)$ of partial sums $s_{n}=\sum_{t=1}^{n}\left(u_{t}-v_{t}\right), n \in \mathbb{N}$, is bounded and $u-v=s-(0, s)$. So

$$
u \succsim v \Longleftrightarrow s-(0, s) \succsim(0,0, \ldots)
$$

By CP, $s \sim(\bar{s}, s)$, where $\bar{s}=\lim _{n \rightarrow \infty} C_{n}(u-v)$ by definition (6). By Add, $s-(0, s) \sim(\bar{s}, s)-$ $(0, s)$. Since

$$
(\bar{s}, s)-(0, s)=(\bar{s}, 0,0, \ldots)
$$

(14) implies

$$
u \succsim v \quad \Longleftrightarrow \quad(\bar{s}, 0,0, \ldots) \succsim(0,0,0, \ldots) .
$$

By reflexivity and SP, $(\bar{s}, 0,0, \ldots) \succsim(0,0,0, \ldots)$ holds if and only if $\bar{s} \geq 0$. With (15), this gives (13).

For TU, if $\sum_{t=1}^{\infty}\left(u_{t}-v_{t}\right)$ is convergent, it is Cesàro-summable to the same sum. That is, $s$ is bounded $(s \in \mathcal{U})$ and $\bar{s}=\sum_{t=1}^{\infty}\left(u_{t}-v_{t}\right)$. By (13), $u \succsim v$ if and only if $\sum_{t=1}^{\infty}\left(u_{t}-v_{t}\right) \geq 0$. 
This leaves us properly equipped for the proof of Theorem 1.

Proof of Theorem 1. For $\delta \in(0,1)$ and $u \in U$, we use the notation $\sigma_{\delta}(u)$ for $\sum_{t=1}^{\infty} \delta^{t-1} u_{t}$. We first show that $\succsim_{\mathrm{LDU}}$ is a social welfare relation. It is reflexive: $\sigma_{\delta}(u-u)=$ $\sigma_{\delta}(0,0, \ldots)=0$ for all $u \in U$ and $\delta \in(0,1)$. It is transitive: let $u, v, w \in \mathcal{U}$ have $u \succsim_{\text {LDU }} v$ and $v \succsim_{\text {LDU }} w$. For each $\delta \in(0,1)$, the discounted sums satisfy

$$
\sigma_{\delta}(u-w)=\sigma_{\delta}(u-v)+\sigma_{\delta}(v-w),
$$

so taking lower limits and using $u \succsim_{\text {LDU }} v$ and $v \succsim_{\text {LDU }} w$ gives

$$
\liminf _{\delta \rightarrow 1^{-}} \sigma_{\delta}(u-w) \geq \liminf _{\delta \rightarrow 1^{-}} \sigma_{\delta}(u-v)+\liminf _{\delta \rightarrow 1^{-}} \sigma_{\delta}(v-w) \geq 0+0=0,
$$

i.e., $u \succsim_{\text {LDU }} w$. We proceed to the axioms:

SP. If $u_{t} \geq v_{t}$ for all $t \in \mathbb{N}$ and $u_{t}>v_{t}$ for some $t \in \mathbb{N}$, the discounted sum $\sigma_{\delta}(u-v)$ is a positive, increasing function of $\delta \in(0,1)$. So $\lim _{\delta \rightarrow 1^{-}} \sigma_{\delta}(u-v)$ exists in $(0,+\infty]$ and $\lim _{\delta \rightarrow 1^{-}} \sigma_{\delta}(v-u)=-\lim _{\delta \rightarrow 1^{-}} \sigma_{\delta}(u-v) \in[-\infty, 0)$. So $u \succ_{\text {LDU }} v$.

Add. For all $u, v, \alpha \in U,(u+\alpha)-(v+\alpha)=u-v$.

CP. Let $u \in U$ have a well defined average $\bar{u}$ and let $c \in \mathbb{R}$. We show that the discounted sum of $d=u-(c, u)=\left(u_{1}-c, u_{2}-u_{1}, u_{3}-u_{2}, \ldots\right)$ satisfies

$$
\lim _{\delta \rightarrow 1^{-}} \sigma_{\delta}(u-(c, u))=\bar{u}-c .
$$

By Frobenius's theorem, it suffices to show that the series $\sum_{t=1}^{\infty} d_{t}$ is Cesàro-summable to $\bar{u}-c$, i.e., that its partial sums $s_{n}=\sum_{t=1}^{n} d_{t}$ satisfy

$$
\frac{s_{1}+\cdots+s_{n}}{n} \rightarrow \bar{u}-c \text { as } n \rightarrow \infty .
$$

But that is easy: the partial sum of the first $n \in \mathbb{N}$ terms is $s_{n}=u_{n}-c$, so

$$
\frac{s_{1}+\cdots+s_{n}}{n}=\frac{u_{1}+\cdots+u_{n}}{n}-c .
$$

Since $\bar{u}$ exists by assumption, the right-hand side tends to $\bar{u}-c$ as $n \rightarrow \infty$. This proves (16). It follows that $(c, u) \sim_{\mathrm{LDU}} u$ if $c=\bar{u}$.

For the second assertion of the theorem, let SWR $\succsim$ satisfy SP, Add, and CP. Let $(u, v) \in \mathcal{D}$. If $d \equiv u-v$ is summable, the Total Utility property (Lemma 2 for both $\succsim_{\text {LDU }}$ and $\succsim)$ implies that $u \succsim v$ if and only if $u \succsim$ LDU $v$.

So assume that $d$ is eventually periodic: there are $p, T \in \mathbb{N}$ with $d_{t+p}=d_{t}$ for all $t \geq T$. For all $t \geq T$, we then have $\bar{d}=\sum_{i=t+1}^{t+p} d_{i} / p$, i.e., $p \bar{d}=\sum_{i=t+1}^{t+p} d_{i}$.

Case 1. If $\bar{d}=0$, then $\sum_{i=t+1}^{t+p} d_{i}=0$ for all $t \geq T$. Then $s_{n}=\sum_{t=1}^{n} d_{t}, n \geq 1$, is eventually periodic, so that $s$ is bounded and $\bar{s}$ is well defined. Since both $\succsim_{\text {LDU }}$ and $\succsim$ satisfy the conditions of Lemma 2,

$$
u \succsim v \quad \Longleftrightarrow \quad \bar{s} \geq 0 \quad \Longleftrightarrow \quad u \succsim_{\mathrm{LDU}} v
$$


Case 2. If $\bar{d} \neq 0$, let us suppose that $\bar{d}>0$; the argument when $\bar{d}<0$ is similar. We show that $u \succ v$ and $u \succ_{\text {LDU }} v$ both hold.

Since $d=u-v$ is bounded, there is an $M \in[0, \infty)$ such that $-M \leq d_{t} \leq M$ for all $t \in \mathbb{N}$. Then also $-M \leq \bar{d} \leq M$. Because $\bar{d}>0$ implies $s_{n} \rightarrow+\infty$, we can choose $N \geq T$ with $s_{N} \geq 2 p M$. We abbreviate $k \in \mathbb{N}$ consecutive zero coordinates by $\underline{0}_{k}$ and define

$$
u^{\prime}=u-\left(\underline{0}_{N}, p \bar{d}, \underline{0}_{p-1}, p \bar{d}, \underline{0}_{p-1}, p \bar{d}, \underline{0}_{p-1}, \ldots\right) .
$$

Since $u-v$ and $\left(\underline{0}_{N}, p \bar{d}, \underline{0}_{p-1}, p \bar{d}, \underline{0}_{p-1}, p \bar{d}, \underline{0}_{p-1}, \ldots\right)$ are eventually periodic with period $p$ and average $\bar{d}$, stream $u^{\prime}-v$ is eventually periodic with period $p$ and average 0 . Arguing as in Case 1, its sequence of partial sums $s_{n}^{\prime}=\sum_{t=1}^{n}\left(u_{t}^{\prime}-v_{t}\right), n \geq 1$, is bounded, i.e., $s^{\prime}=\left(s_{1}^{\prime}, s_{2}^{\prime}, \ldots\right) \in \mathcal{U}$, and eventually periodic with period $p$. We claim that $s_{n}^{\prime} \geq 0$ for all $n>N$. By periodicity, it suffices to show that $s_{N+k}^{\prime} \geq 0$ for all $k=1, \ldots, p$. By construction,

$$
s_{N+k}^{\prime}=s_{N}-p \bar{d}-\sum_{m=1}^{k}\left(u_{N+m}-v_{N+m}\right) \geq 2 p M-p M-k M \geq 0 .
$$

Since $s_{n}^{\prime} \geq 0$ for all $n>N, \bar{s}^{\prime} \geq 0$. By CP, $s^{\prime} \sim\left(\bar{s}^{\prime}, s^{\prime}\right)$, so $s^{\prime} \succsim\left(0, s^{\prime}\right)$ by SP. By Add, $u^{\prime} \succsim v$. By SP, $u \succ u^{\prime}$. By transitivity, $u \succ v$. Since $\succsim_{\text {LDU }}$ also satisfies Add, SP, and CP, the same arguments show that $u \succ_{\text {LDU }} v$.

\section{A.3 Proof of Lemma 1}

We prove Lemma 1 slightly more generally, for all real sequences $d=\left(d_{1}, d_{2}, \ldots\right)$ whose discounted sum $\sigma_{\delta}(d)$ is well defined for each $\delta \in(0,1)$. Let $s_{n}=\sum_{t=1}^{n} d_{t}, n \in \mathbb{N}$. In (7), note that all upper/lower limits are well defined in $\mathbb{R} \cup\{-\infty,+\infty\}$ and that the second inequality holds: all infima and suprema are taken over nonempty sets. The first inequality in (7) implies the third using a sign change: $\lim \sup _{\delta \rightarrow 1^{-}} \sigma_{\delta}(d)=-\liminf _{\delta \rightarrow 1^{-}} \sigma_{\delta}(-d)$, $\limsup _{n \rightarrow \infty} C_{n}(d)=-\liminf _{n \rightarrow \infty} C_{n}(-d)$. So it suffices to prove the first inequality:

$$
\liminf _{n \rightarrow \infty} C_{n}(d) \leq \liminf _{\delta \rightarrow 1^{-}} \sigma_{\delta}(d) .
$$

Summation by parts, first for the sequence $d$ and then for its partial sums, gives

$$
\begin{aligned}
\sum_{n=1}^{\infty} \delta^{n-1} d_{n} & =d_{1}+\sum_{n=2}^{\infty} \delta^{n-1}\left(s_{n}-s_{n-1}\right) \\
& =(1-\delta) \sum_{n=1}^{\infty} \delta^{n-1} s_{n} \\
& =(1-\delta)^{2} \sum_{n=1}^{\infty} \delta^{n-1}\left(s_{1}+\cdots+s_{n}\right)
\end{aligned}
$$


So, recalling from (6) that $C_{n}(d)=\left(s_{1}+\cdots+s_{n}\right) / n$, the discounted sum equals

$$
\sigma_{\delta}(d)=\sum_{n=1}^{\infty} \delta^{n-1} d_{n}=(1-\delta)^{2} \sum_{n=1}^{\infty} \delta^{n-1} n C_{n}(d) .
$$

Distinguish three cases. First, if $\liminf _{n \rightarrow \infty} C_{n}(d)=+\infty$, then (17) and the equality

$$
\sum_{n=1}^{\infty} n \delta^{n-1}=\frac{1}{(1-\delta)^{2}}
$$

immediately give that also $\liminf _{\delta \rightarrow 1^{-}} \sigma_{\delta}(d)=+\infty$. Second, if $\liminf _{n \rightarrow \infty} C_{n}(d)=-\infty$, the inequality liminf $\operatorname{in}_{n \rightarrow \infty} C_{n}(d) \leq \liminf _{\delta \rightarrow 1^{-}} \sigma_{\delta}(d)$ holds trivially, since both lower limits are well defined. Finally, suppose that $\liminf _{n \rightarrow \infty} C_{n}(d)$ is finite. For $\lambda \in \mathbb{R}$, (17) and (18) give

$$
\sum_{n=1}^{\infty} \delta^{n-1} d_{n}-\lambda=\left(1-\delta^{2}\right) \sum_{n=1}^{\infty} \delta^{n-1} n\left(C_{n}(d)-\lambda\right) .
$$

Take $\lambda=\liminf _{n \rightarrow \infty} C_{n}(d)$. By definition of $\lambda$, for each $\varepsilon>0$ there is a $T$ such that $C_{n}(d)-$ $\lambda \geq-\varepsilon$ for all $n>T$. Then

$$
\begin{aligned}
\sum_{n=1}^{\infty} \delta^{n-1} d_{n}-\lambda & \geq\left(1-\delta^{2}\right) \sum_{n=1}^{T} \delta^{n-1} n\left(C_{n}(d)-\lambda\right)-\varepsilon\left(1-\delta^{2}\right) \sum_{n=T+1}^{\infty} \delta^{n-1} n \\
& \geq\left(1-\delta^{2}\right) \sum_{n=1}^{T} \delta^{n-1} n\left(C_{n}(d)-\lambda\right)-\varepsilon .
\end{aligned}
$$

The first term in (19) tends to 0 as $\delta \rightarrow 1^{-}$, so $\liminf _{\delta \rightarrow 1^{-}} \sigma_{\delta}(d)-\lambda \geq-\varepsilon$. Since $\varepsilon>0$ was arbitrary, $\liminf _{\delta \rightarrow 1^{-}} \sigma_{\delta}(d) \geq \lambda=\liminf _{n \rightarrow \infty} C_{n}(d)$.

\section{A.4 Proof of Theorem 2}

TU. If $u-v$ is summable, then $\sum_{t=1}^{\infty}\left(u_{t}-v_{t}\right)=\lim _{\delta \rightarrow 1^{-}} \sigma_{\delta}(u-v)$ by Abel's theorem; cf. Duren (2012, p. 76). (An indirect proof is given by Lemma 2.)

Ano. If $v \in U$ is obtained from $u \in U$ by permuting two coordinates, then $\sum_{t=1}^{\infty}\left(u_{t}-v_{t}\right)=0$. So Ano follows from TU.

Stat. For all $u, v \in U, \liminf _{\delta \rightarrow 1^{-}} \sigma_{\delta}(u-v)=\left(u_{1}-v_{1}\right)+\liminf _{\delta \rightarrow 1^{-}} \sigma_{\delta}\left(\left(u_{2}, u_{3}, \ldots\right)-\right.$ $\left.\left(v_{2}, v_{3}, \ldots\right)\right)$.

Cont. Let $u, v \in \mathcal{U}$ and let $N \in \mathbb{N}$ be such that $u_{[n]} \succ_{\text {LDU }} v_{[n]}$ for all $n \geq N$. Since $u_{[n]}$ and $v_{[n]}$ are summable, $s_{n}=\sum_{t=1}^{n}\left(u_{t}-v_{t}\right)>0$ for all $n \geq N$ by TU. By Lemma 1 , $\liminf _{\delta \rightarrow 1^{-}} \sigma_{\delta}(u-v) \geq 0$, i.e., $u \succsim_{\text {LDU }} v$.

\section{A.5 Proof of Theorem 3}

(i) Since $\succsim_{\text {LDu }}$ satisfies SP, Ano, and Add, it extends $\succsim_{\text {вм }}$; see Basu and Mitra (2007, Theorem 1) or Jonsson and Voorneveld (2015, Theorem 6). 
(ii) By definition, $\succsim_{\mathrm{G}}$ weakly extends $\succsim_{\mathrm{W}}$. To see that $\succsim_{\text {LDU }}$ extends $\succsim_{\mathrm{G}}$, let $u, v \in$ $u$ have $u \succsim_{\mathrm{G}} v$ : the partial sums $s_{n}=\sum_{t=1}^{n}\left(u_{t}-v_{t}\right)$ of $u-v$ satisfy $\liminf _{n \rightarrow \infty} s_{n} \geq 0$. Consequently,

$$
\liminf _{n \rightarrow \infty} C_{n}(u-v)=\liminf _{n \rightarrow \infty} \frac{s_{1}+\cdots+s_{n}}{n} \geq 0 .
$$

By Lemma 1, also $\lim \inf _{\delta \rightarrow 1^{-}} \sigma_{\delta}(u-v) \geq 0$. That is, $u \succsim_{\text {LDU }} v$.

Finally, (iii) was illustrated in Example 1, and (iv) was illustrated in Example 3.

\section{REFERENCES}

Asheim, Geir B. (2010), "Intergenerational equity." Annual Review of Economics, 2, 197222. [23]

Asheim, Geir B. and Kuntal Banerjee (2010), "Fixed-step anonymous overtaking and catching-up.” International Journal of Economic Theory, 6, 149-165. [26]

Asheim, Geir B., Claude d'Aspremont, and Kuntal Banerjee (2010), "Generalized timeinvariant overtaking." Journal of Mathematical Economics, 46, 519-533. [21, 23, 24, 26]

Asheim, Geir B. and Bertil Tungodden (2004), "Resolving distributional conflicts between generations." Economic Theory, 24, 221-230. [25, 26]

Banerjee, Kuntal (2006), "On the extension of the utilitarian and Suppes-Sen social welfare relations to infinite utility streams." Social Choice and Welfare, 27, 327-339. [26]

Banerjee, Kuntal and Tapan Mitra (2007), "On the impatience implications of Paretian social welfare functions.” Journal of Mathemetical Economics, 43, 236-248. [20]

Basu, Kaushik and Tapan Mitra (2003), "Aggregating infinite utility streams with intergenerational equity: The impossibility of being Paretian." Econometrica, 71, 1557-1563. [20]

Basu, Kaushik and Tapan Mitra (2007), "Utilitarianism for infinite utility streams: A new welfare criterion and its axiomatic characterization." Journal of Economic Theory, 133, $350-373 .[20,23,25,26,34]$

Bishop, Christopher J., Eugene A. Feinberg, and Junyu Zhang (2014), "Examples concerning Abel and Cesàro limits." Journal of Mathematical Analysis and Applications, 420, 1654-1661. [20, 25, 28, 29]

Brock, William A. (1970), "An axiomatic basis for the Ramsey-Weizsäcker overtaking criterion.” Econometrica, 38, 927-929. [21, 25]

Diamond, Peter A. (1965), “The evaluation of infinite utility streams.” Econometrica, 33, 170-177. [20]

Duren, Peter L. (2012), Invitation to Classical Analysis, volume 17 of Pure and Applied Undergraduate Texts. American Mathematical Society. [23, 28, 34]

Dutta, Bhaskar (2008), "Some remarks on the ranking of infinite utility streams." In Arguments for a Better World: Essays in Honor of Amartya Sen, Volume I: Ethics, Welfare, 
and Measurement (Kaushik Basu and Ravi Kanbur, eds.), 136-147, Chapter 8, Oxford University Press. [21, 26]

Dutta, Prajit K. (1991), "What do discounted optima converge to? A theory of discount rate asymptotics in economic models." Journal of Economic Theory, 55, 64-94. [20]

Fleurbaey, Marc and Philippe Michel (2003), "Intertemporal equity and the extension of the Ramsey criterion.” Journal of Mathematical Economics, 39, 777-802. [21, 26]

Frobenius, Georg (1880), “Über die Leibnizsche Reihe.” Journal für die reine und angewandte Mathematik, 89, 262-264. [21]

Gale, David (1967), "On optimal development in a multi-sector economy." Review of Economic Studies, 34, 1-18. [20, 26]

Heal, Geoffrey (2005), "Intertemporal welfare economics and the environment." In Handbook of Environmental Economics, Vol. 3 (Karl-Göran Mäler and Jeffrey R. Vincent, eds.), 1105-1145, Chapter 21, Elsevier. [21, 23, 26]

Jonsson, Adam and Mark Voorneveld (2015), "Utilitarianism on infinite utility streams: Summable differences and finite averages." Economic Theory Bulletin, 3, 19-31. [25, 26, $34]$

Koopmans, Tjalling C. (1960), "Stationary ordinal utility and impatience." Econometrica, 28, 287-309. [19, 20, 24]

Koopmans, Tjalling C., Peter A. Diamond, and Richard E. Williamson (1964), "Stationary utility and time perspective." Econometrica, 32, 82-100. [23]

Lauwers, Luc (1995), “Time-neutrality and linearity.” Journal of Mathematical Economics, 24, 347-351. [21, 26]

Lauwers, Luc (1997), "Infinite utility: Insisting on strong monotonicity." Australasian Journal of Philosophy, 75, 222-233. [26]

Lauwers, Luc (2010), "Ordering infinite utility streams comes at the cost of a nonRamsey set.” Journal of Mathematical Economics, 46, 32-37. [20]

Liggett, Thomas M. and Steven A. Lippman (1969), "Stochastic games with perfect information and time average payoff." SIAM Review, 11, 604-607. [20, 28]

Lippman, Steven A. (1969), "Letter to the editor-Criterion equivalence in discrete dynamic programming." Operations Research, 17, 920-922. [20, 25]

Pigou, Arthur (1920), The Economics of Welfare. Macmillan, London. [19]

Ramsey, Frank P. (1928), "A mathematical theory of saving.” The Economic Journal, 38, 543-559. [19, 20]

Sennott, Linn I. (1999), Stochastic Dynamic Programming and the Control of Queueing Systems. Wiley Series in Probability and Statistics. John Wiley \& Sons, New York. [20, 25]

Sidgwick, Henry (1907), The Methods of Ethics, seventh edition. Macmillan, London. [19] 
Svensson, Lars-Gunnar (1980), “Equity among generations.” Econometrica, 48, 12511256. [20]

von Weizsäcker, Carl Christian (1965), "Existence of optimal programs of accumulation for an infinite time horizon." Review of Economic Studies, 32, 85-104. [20, 26]

Zame, William R. (2007), “Can intergenerational equity be operationalized?” Theoretical Economics, 2, 187-202. [20]

Co-editor Faruk Gul handled this manuscript.

Manuscript received 26 April, 2014; final version accepted 30 January, 2017; available online 14 February, 2017. 